\title{
Formation of Titan in Saturn's subnebula: constraints from Huygens probe measurements
}

\author{
Y. Alibert ${ }^{1}$ and O. Mousis ${ }^{2}$ \\ 1 Physikalisches Institut, University of Bern, Sidlerstrasse 5, 3012 Bern, Switzerland \\ e-mail: yann. alibert@space.unibe.ch \\ 2 Observatoire de Besançon, CNRS-UMR 6091, BP 1615, 25010 Besançon Cedex, France
}

Received 15 September 2006 / Accepted 12 December 2006

\begin{abstract}
We present an evolutionary turbulent model of the Saturn's subnebula consistent with recent core accretion formation models of Saturn. Our calculations are similar to those conducted in the case of the Jovian subnebula, and take into account the vertical structure of the disk, as well as the evolution of its surface density, as given by an $\alpha$-disk model. Using the thermodynamic conditions of our model, we calculate the evolution of the $\mathrm{CO}_{2}: \mathrm{CO}: \mathrm{CH}_{4}$ and $\mathrm{N}_{2}: \mathrm{NH}_{3}$ molar mixing ratios in the subnebula. We thus show that the carbon and nitrogen homogeneous gas-phase chemistry is inhibited in the subnebula. We also consider the role played by Fischer-Tropsch catalysis in the gas-phase conversions of $\mathrm{CO}$ and $\mathrm{CO}_{2}$ into $\mathrm{CH}_{4}$. We demonstrate that, even if a catalytically active zone is likely to exist in the early Saturn's subnebula, it does not alter the composition of volatiles ultimately trapped in the forming solids. We study two different formation scenarios of Titan. In each scenario, we provide observational tests that are compared with measurements made by the Huygens probe. In the first scenario, Titan is formed in a late and cold subnebula from planetesimals produced in Saturn's feeding zone that have been preserved from vaporization. In the second scenario, Titan is formed in a balmy and early subnebula. We show that the first scenario predicts a $\mathrm{CO}: \mathrm{CH}_{4}$ molar mixing ratio orders of magnitude larger than the observed one in the atmosphere of Titan, and requires strong variations of water abundance in the solar nebula on short lengthscales, whose origin is not explained. On the contrary, the second scenario does not require such large variations of the abundance of water, and predicts abundances of volatile species in Titan similar to the observed ones.
\end{abstract}

Key words. planets and satellites: formation - planets and satellites: general

\section{Introduction}

The exploration of Titan by the Cassini-Huygens spacecraft has shed new light on its formation, as well as on the origin of Saturn's satellite system. The measurements made by the Gas Chromatograph Mass Spectrometer (GCMS) aboard the Huygens probe have confirmed that the atmosphere of Titan is dominated by $\mathrm{N}_{2}$ and $\mathrm{CH}_{4}$, with only a tiny abundance of $\mathrm{CO}$, in agreement with previous determinations (see Gautier \& Raulin 1997). The abundance of Ar was also found to be very low, those of $\mathrm{Kr}$ and $\mathrm{Xe}$ were too weak to be determined (Niemann et al. 2005). These latter measurements are at odd with the detection of noble gases in the atmospheres of Mars and Venus (Biemann et al. 1976; Donahue et al. 1981), in some meteorites (Marty et al. 2006), and in the atmosphere of Jupiter, where their abundances are found to be over-solar (Owen et al. 1999). Moreover, thermochemical calculations, using different models of Saturn's subnebula, also predict noble gas abundances greater than, or equal to solar ones in Titan (Prinn \& Fegley 1981; Mousis et al. 2002, hereafter MGB02).

Using evolutionary turbulent models of the Saturn's subnebula, MGB02 derived the composition of planetesimals that took part in the formation of Titan, assuming that volatile species (noble gases, as well as $\mathrm{NH}_{3}, \mathrm{CH}_{4}$ ) were trapped as hydrates or clathrate hydrates during the cooling of the subnebula. They concluded that $\mathrm{Xe}: \mathrm{C}$ should be over-solar, in contradiction to recent measurements of the Huygens probe (Niemann et al. 2005). However, MGB02 assumed that Saturn's subnebula was in equilibrium (the accretion rate being constant over the whole subnebula) during its cooling. Moreover, they assumed that it was a closed system, neglecting the fact that Saturn accreted gas and gas coupled material during a substantial fraction of the subnebula's lifetime (Lubow et al. 1999; Magni \& Coradini 2004). This implies that the accretion rate in the subnebula must, contrary to the work of MGB02, be consistent with formation models of Saturn itself.

In two recent papers, Alibert et al. (2005d, hereafter AMB05) and Mousis \& Alibert (2006, hereafter MA06) have presented models of the Jovian subnebula structure and evolution, consistent with the last phases of Jupiter formation. This model was derived from the concept advocated both by Stevenson (2001) and Canup \& Ward (2002): the so called gasstarved disk model (see also Canup \& Ward 2006). In this model, the subnebula appears during the late phases of the giant planet formation, and is fed at its outer edge by gas and gas-coupled material originating from the solar nebula.

In this paper, we calculate a model of Saturn's subnebula, using a method similar to the one of AMB05 and MA06. This formalism allowing to calculate subnebula models consistent with the end phases of Saturn's formation, thus avoiding the limitations of the model of MGB02 described above. As in the case of the Jovian subnebula, Saturn's subnebula evolves in two phases. During the first one, when the solar nebula is still present, the subnebula is quite massive, the temperature being high, in particular close to the planet. When the solar nebula has disappeared, the subnebula rapidly cools down, its material being accreted by 
the central planet. The structure and evolution of the subnebula model thus appear to be very different from the one used in MGB02.

Using the thermodynamical conditions derived from our model, we consider the formation of Titan, the most massive satellite of Saturn. As we shall see in this paper, interpreting the GCMS measurements aboard the Huygens probe can be used to derive constraints on Titan's formation, from planetesimals ${ }^{1}$ whose origin can be twofold. Titan's building blocks may have been produced in the solar nebula, and be further carried away by gas flowing from the solar nebula to Saturn's subnebula. They may also have been directly formed from ices produced inside the cooling subnebula itself. According to the considered origin, the composition of planetesimals may differ, due to nitrogen and carbon gas-phase chemistry in the initially warm subnebula.

As stated above, the deficiency of Titan's atmosphere both in $\mathrm{CO}$ and noble gases is difficult to explain in the framework of available models. The low amount of $\mathrm{CO}$ could result from chemical reactions between $\mathrm{C}$ bearing species, as initially proposed by Prinn \& Fegley (1981) $\left(\mathrm{CO}, \mathrm{CH}_{4}\right.$ and $\mathrm{CO}_{2}$ for the most important ones). Therefore, it is important to study the evolution of the $\mathrm{CO}_{2}: \mathrm{CO}: \mathrm{CH}_{4}$ and $\mathrm{N}_{2}: \mathrm{NH}_{3}$ molar mixing ratios in the subnebula gas-phase. Both homogeneous and heterogeneous gasphase chemistries could convert part of the $\mathrm{CO}$ and $\mathrm{CO}_{2}$ initially present in the solar nebula to $\mathrm{CH}_{4}$. MGB02 concluded from their study that the evolution of the $\mathrm{CO}: \mathrm{CH}_{4}$ and $\mathrm{N}_{2}: \mathrm{NH}_{3}$ molar mixing ratios was inhibited in their subnebula model. However, the thermodynamic conditions in our model differ largely from those in MGB02. This prompted us to reexamine the chemistry of $\mathrm{CO}: \mathrm{CH}_{4}$ and $\mathrm{N}_{2}: \mathrm{NH}_{3}$ in our model. Moreover, $\mathrm{CO}_{2}$ was not considered in MGB02, but may represent a substantial part of the $\mathrm{C}$ bearing volatiles in Saturn's subnebula. Indeed, $\mathrm{CO}_{2}$ has been detected in Phoebe (Clark et al. 2005), an irregular satellite believed to have been formed in the solar nebula and further captured by Saturn. This points toward the presence of $\mathrm{CO}_{2}$ in the solar nebula around 10 AU. Finally, MGB02 did not consider the effect of heterogeneous gas-phase chemistry resulting from Fischer-Tropsch catalysis (due to the presence of metal grains in the subnebula) on the evolution of the carbon chemistry. In fact, Fischer-Tropsch catalysis in Saturn's subnebula converts $\mathrm{CO}$ and $\mathrm{CO}_{2}$ to $\mathrm{CH}_{4}$, and could be at the origin of the deficiency of CO in Titan. We therefore extend the calculations of MGB02 to the heterogeneous chemistry of $\mathrm{CO}_{2}: \mathrm{CO}: \mathrm{CH}_{4}$ using the thermodynamic conditions of our subnebula model and considering the recent laboratory determinations of the Fischer-Tropsch reaction rates by Sekine et al. (2005). Our calculations thus allow us to determine the composition of ices incorporated in planetesimals formed in Saturn's subnebula, or migrating inside it. Our calculations show that both homogeneous and heterogeneous gas-phase chemistries do not affect the composition of planetesimals that may have taken part to the formation of Titan. Conversion timescales in Saturn's subnebula are too long to efficiently convert $\mathrm{CO}$ to $\mathrm{CH}_{4}$. On the other hand, we show that, even if Fischer-Tropsch catalysis could have been active in Saturn's subnebula, its products were accreted by the planet and not incorporated in forming planetesimals. Therefore, neither of the afore mentioned gas-phase chemical processes can explain the low abundance of CO in Titan's atmosphere.

We consider two main scenarios for Titan's formation. The first scenario invokes the formation of Titan in a late and cold subnebula from planetesimals formed in the solar nebula,

\footnotetext{
1 In this paper, solids produced in the solar nebula or Saturn's subnebula are called planetesimals whatever their origin.
}

namely in Saturn's feeding zone. In this scenario, we examine the influence of an ad hoc deficiency of water in the formation zone of Saturn on the composition of the planetesimals that were ultimately accreted by Titan (similarly to what has been proposed by Hersant et al. 2004 in the case of Saturn; see also Hersant et al. 2005). In the second scenario, we assume that Titan formed in a balmy subnebula, and do not invoke any deficiency of water in the formation region of planetesimals. Instead, we propose that the planetesimals accreted by Titan have suffered a partial vaporization during their migration inside the subnebula. We compare the results of these two models with the GCMS measurements aboard the Huygens probe, and show that the first one implies a $\mathrm{CO}: \mathrm{CH}_{4}$ ratio orders of magnitude larger than that inferred from current observations of Titan. On the contrary, the second scenario, although very simplified in our calculations, is compatible with the recent determination of $\mathrm{CO}$ and noble gas abundances in Titan's atmosphere.

The paper is organized as follows: in Sect. 2 we present our model of the Saturn's subnebula. The structure and evolution of the subdisk are controlled by the last phases of Saturn's formation, as derived in the model of Alibert et al. (2005c). In Sect. 2.3 we calculate the thermodynamic properties inside the subnebula. In Sect. 3, we study the evolution of the $\mathrm{CO}_{2}: \mathrm{CO}: \mathrm{CH}_{4}$ and $\mathrm{N}_{2}: \mathrm{NH}_{3}$ molar mixing ratios and we deduce in Sect. 4 the composition of planetesimals that may have taken part to the formation of Titan. In Sect. 5, we investigate the possible formation scenarios of Titan, using the latest results obtained by the Huygens probe.

\section{Model of Saturn's subnebula}

\subsection{Origin and formation}

Our subnebula model is based on the gas-starved disk model (Stevenson 2001; Canup \& Ward 2002, 2006), extended to timedependent accretion rates. The model is briefly recalled here and more details can be found in the original papers by Stevenson (2001), Canup \& Ward (2002), and in AMB05. At the beginning of Saturn's formation process, its total radius is equal to its Hill's radius, and no subnebula can exist. At the end of the formation process, the cooling rate of the planet's envelope is such that its radius shrinks faster than the disk can supply additional gas. Accretion of gas now proceeds through streamers which eventually collide and form a circum-planetary disk (Lubow et al. 1999). When the radius of the proto-planet becomes small enough, a subnebula emerges from the contracting atmosphere (Coradini et al. 1995). This subnebula is fed by gas and gas-coupled solids accreted from the solar nebula. The accretion rate of gas from the solar nebula to the subnebula is therefore directly driven by the planet's accretion rate of gas, derived in the framework of our giant planet formation model (Alibert et al. 2005c).

\subsection{Structure and evolution}

The structure of the subnebula is calculated in the framework of the $\alpha$-disk model of Shakura \& Sunyaev (1973), by solving the diffusion equation:

$$
\frac{\mathrm{d} \Sigma}{\mathrm{d} t}=\frac{3}{r} \frac{\partial}{\partial r}\left[r^{1 / 2} \frac{\partial}{\partial r} \tilde{v} \Sigma r^{1 / 2}\right]
$$

where $\Sigma$ is the surface density in gas phase, $r$ is the distance to the planet and $\tilde{v}$ the mean viscosity (the mean being taken along the 
Table 1. Thermodynamic parameters of Saturn's subnebula.

\begin{tabular}{lc}
\hline \hline Thermodynamic \\
parameters \\
\hline Mean mol. weight $(\mathrm{g} /$ mole $)$ & 2.4 \\
$\alpha$ & $2 \times 10^{-4}$ \\
Disk's inner radius $\left(R_{\text {Sat }}\right)$ & 4 \\
Initial disk's radius $\left(R_{\text {Sat }}\right)$ & 200 \\
Initial disk's mass $\left(M_{\text {Sat }}\right)$ & $7 \times 10^{-3}$ \\
Initial accretion rate $\left(M_{\text {Sat }} / \mathrm{yr}\right)$ & $1.8 \times 10^{-7}$ \\
\hline
\end{tabular}

vertical axis). In this equation, the origin of time in the subdisk is arbitrarily taken when the planet has already accreted $\sim 70 \%$ of its total mass. However, during the first phase of its evolution, the subnebula is in equilibrium, the accretion rate being constant for all radii. Hence, the origin of time has no great importance.

The subnebula's viscosity used in Eq. (1) is calculated by determining the vertical structure of the subnebula for each distance from the planet. This vertical structure is determined as follows (see AMB05 and Alibert et al. 2005a, for details): for each distance from Saturn, we solve the equation for hydrostatic equilibrium, together with the energy equation and the diffusion equation for the radiative flux. The local viscosity is therefore calculated using the standard Shakura \& Sunyaev (1973) $\alpha$-parametrization $v=\alpha C_{\mathrm{s}}^{2} / \Omega$ where the speed of sound $C_{\mathrm{s}}$ is determined by the vertical temperature profile, and $\Omega^{2}=$ $G M_{\text {planet }} / r^{3}$. We use for the calculations presented here the same $\alpha$ value as in the case of the Jovian subnebula, namely $\alpha=2 \times$ $10^{-4}$. Using this procedure, we calculate the mean viscosity $\tilde{v}$ as a function of the gas surface density $\Sigma$ and the distance from the planet $r$. Other parameters of our model are summarized in Table 1 . These calculations also give as a by-product the relations between the midplane pressure, temperature and surface density that will be used to calculate the evolution of the gasphase chemistry.

Two boundary conditions, as well as an initial condition, are necessary to solve the diffusion equation. For the initial condition, we assume that at $t=0$, the subnebula is in equilibrium, the accretion rate in the subnebula being uniform (by definition of an equilibrium model) and given by the accretion rate of gas from the solar nebula toward the forming planet. The inner boundary condition is taken at $4 R_{\text {Sat }}$ (radius of Saturn). We assume that, for radii below this value, the subdisk is always in equilibrium and instantaneously adapts to the conditions given by its outer edge (see Papaloizou \& Terquem 1999; Alibert et al. 2005a).

The outer boundary condition varies depending upon the presence of the solar nebula. When the solar nebula has not dissipated (that we call phase 1 of the subnebula evolution), the subnebula is fed at its outer radius by gas and gas-coupled solids originating from the solar nebula. We determine the accretion rate of gas at the outer radius of the subnebula by using the results of the Saturn formation model (Alibert et al. 2005c): the accretion rate from the nebula to the subnebula is the planet's accretion rate of gas calculated during the late phase of its formation. Based on hydrodynamical simulations (Lubow et al. 1999), the outer radius of the subnebula was found to be around $1 / 5$ Hill's radius in the case of Jupiter. In the absence of comparable calculations for Saturn, we adopt the same external radius of $1 / 5$ Hill's radius in our model (around $200 R_{\text {Sat }}$ ). During phase 1 of the subnebula evolution, the accretion rate of gas is constant inside the whole subdisk, which is in equilibrium. This justifies a posteriori the use of an equilibrium model as an initial model

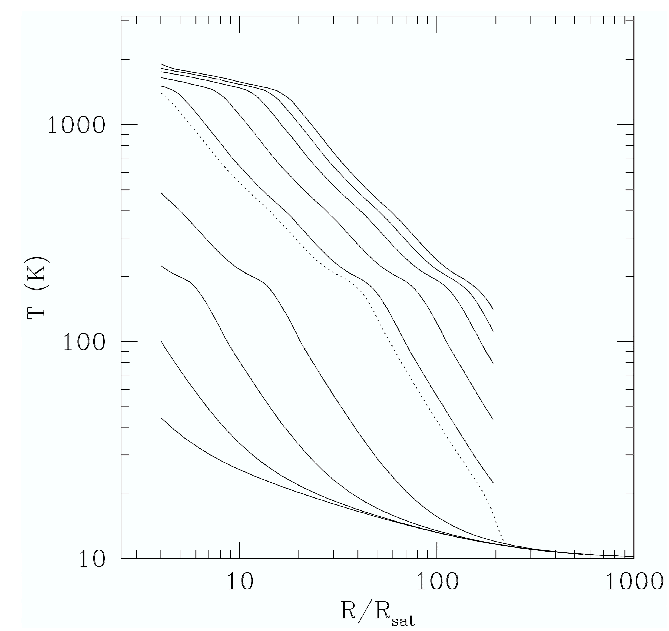

Fig. 1. Temperature inside the subnebula, as a function of the distance from Saturn (in Saturn's radii). The solid lines are plotted, from top to bottom, at $t=0 \mathrm{Myr}$ (corresponding to the time when Saturn has accreted $70 \%$ of its final mass), $0.1 \mathrm{Myr}, 0.2 \mathrm{Myr}, 0.3 \mathrm{Myr}, 0.35 \mathrm{Myr}$, $0.4 \mathrm{Myr}, 0.5 \mathrm{Myr}, 0.6 \mathrm{Myr}$ and $0.7 \mathrm{Myr}$. The switch from phase 1 to phase 2 of the subnebula evolution occurs at $0.36 \mathrm{Myr}$. The dotted line gives the temperature 2000 years after the beginning of phase 2, and illustrates the very rapid evolution of the subnebula during this stage. During phase 1 (first five curves), the outer radius of the subnebula is equal to $200 R_{\text {Sat }}$.

of the subnebula. This phase lasts until the solar nebula has disappeared, namely at $t=0.36 \mathrm{Myr}$, according to our model. The time evolution of the subnebula is therefore directly governed by the last phase of Saturn's formation.

After $t=0.36 \mathrm{Myr}$, the solar nebula has vanished, and the subnebula enters its second phase of evolution. The subnebula evolves due to accretion of its own material onto the planet, and expands due to angular momentum conservation. The accretion rate as a function of the distance to Saturn is no longer constant. Hence, one cannot use an equilibrium model (i.e. with an uniform accretion rate throughout all the subnebula) and the diffusion equation has to be solved. During this phase, the outer radius of the subdisk is allowed to freely expand. The surface density is fixed to 0 at $1050 R_{\text {Sat }}$, corresponding to Saturn's Hill radius. More technical details on the calculation of the structure and evolution of the subnebula can be found in AMB05.

\subsection{Thermodynamic conditions inside Saturn's subnebula}

The calculation of the vertical structure of the disk, used to determine the mean viscosity inside the subnebula, gives as a byproduct the midplane thermodynamic conditions, presented in Figs. 1-3 as a function of the distance from Saturn, for different times. Water is in vapor phase at $t=0$ in the subnebula at radii lower than $\sim 180 R_{\text {Sat }}$, and $T, P$, and $\Sigma$ decrease with time and with the distance from Saturn. The cooling of the subnebula results in the inward propagation of a water condensation front that reaches the present orbit of Titan $\left(\sim 20 R_{\text {Sat }}\right)$ at $t=0.38 \mathrm{Myr}$.

During the evolution of the subnebula, the temperature reaches very low values (of the order of $\sim 20 \mathrm{~K}$ ), after $\sim 0.35 \mathrm{Myr}$ at the present day orbit of Phoebe $\left(\sim 210 R_{\text {Sat }}\right.$ close to the external radius of our model of the subnebula during phase 1) and $\sim 0.7 \mathrm{Myr}$ at the present day orbit of Titan. 


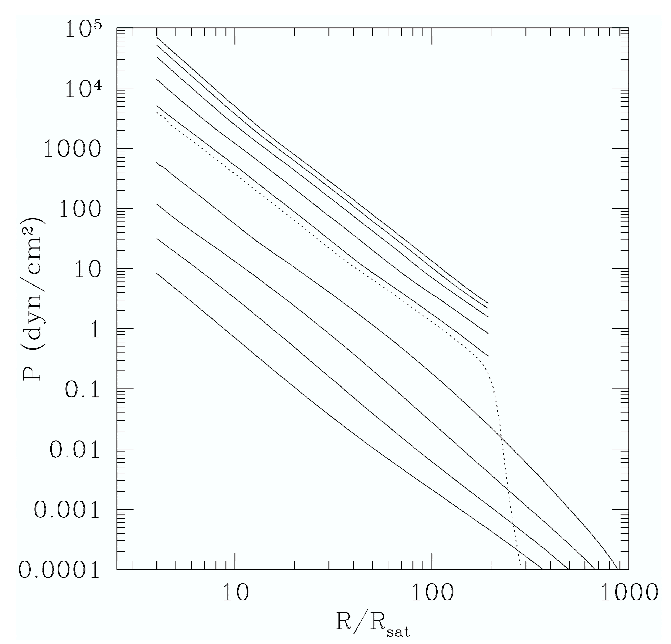

Fig. 2. Log of pressure at different epochs in the Saturn's subnebula midplane. Epochs are the same as in Fig. 1.

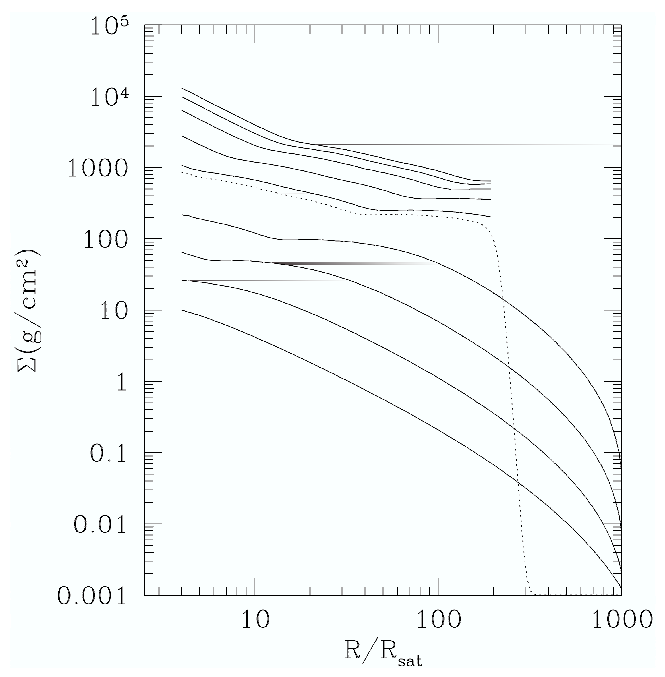

Fig. 3. Log of gas surface density at different epochs in the Saturn's subnebula midplane. Epochs are the same as in Fig. 1.

\section{Chemistry of $\mathrm{C}$ - and $\mathrm{N}$-bearing volatiles in the subnebula}

Since ices were initially all vaporized in Saturn's subnebula beyond $\sim 180 R_{\text {Sat }}$ (see Fig. 4 ), gas-phase reactions may occur for major $\mathrm{C}$ and $\mathrm{N}$ volatiles in this environment. This may have some important effects on the composition of planetesimals that formed from ices produced in the subnebula (see Sect. 4.2). We first examine the homogeneous gas-phase reactions of $\mathrm{C}$ and $\mathrm{N}$ volatiles in Saturn's subnebula, following the approach proposed by MGB02. We also investigate the catalytic effects on the gas-phase reactions induced by the presence of iron particles mixed with hydrogen gas in Saturn's subnebula, using the experimental data of Sekine et al. (2005) and following the approach proposed by Mousis et al. (2006b) for the Jovian subnebula.

\subsection{Homogeneous gas-phase chemistry}

Following Prinn \& Fegley (1989), the net reactions relating CO, $\mathrm{CH}_{4}, \mathrm{CO}_{2}, \mathrm{~N}_{2}$ and $\mathrm{NH}_{3}$ in a gas dominated by $\mathrm{H}_{2}$ are

$\mathrm{CO}+\mathrm{H}_{2} \mathrm{O}=\mathrm{CO}_{2}+\mathrm{H}_{2}$

$\mathrm{CO}+3 \mathrm{H}_{2}=\mathrm{CH}_{4}+\mathrm{H}_{2} \mathrm{O}$

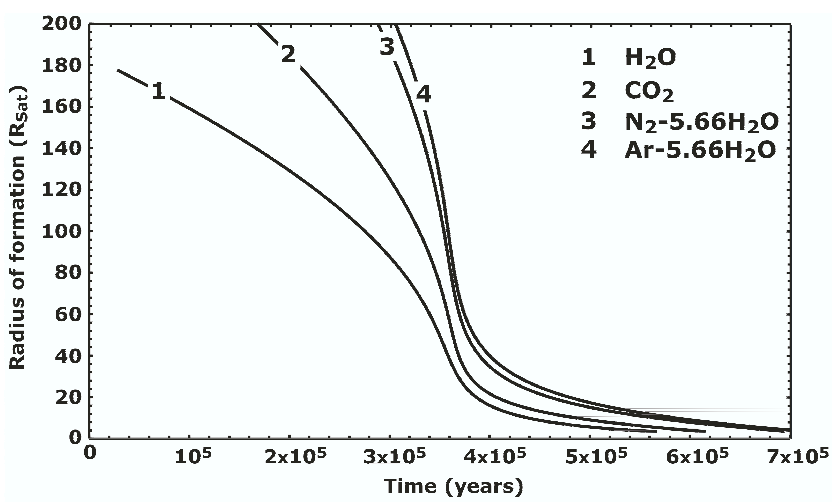

Fig. 4. Radii of formation of $\mathrm{H}_{2} \mathrm{O}$ ice, $\mathrm{CO}_{2}$ pure condensate, $\mathrm{N}_{2}$ and Ar clathrate hydrates in Saturn's subnebula as a function of time. Radii of formation of $\mathrm{NH}_{3}-\mathrm{H}_{2} \mathrm{O}$ hydrate and $\mathrm{H}_{2} \mathrm{~S}, \mathrm{Xe}, \mathrm{CH}_{4}, \mathrm{CO}$ and $\mathrm{Kr}$ clathrate hydrates are not represented but are within curves 1 and 3 .

$\mathrm{N}_{2}+3 \mathrm{H}_{2}=2 \mathrm{NH}_{3}$

which all proceed to the right with decreasing temperature at constant pressure. Reaction (2) has been studied by Talbi \& Herbst (2002) who demonstrated that its rate coefficient is negligible, even at temperature as high as $2000 \mathrm{~K}$. Such a high temperature range is only reached at distances far from Saturn and at early epochs in Saturn's subnebula (see Fig. 1). As a result, the amount of carbon species produced through this reaction is not significant during the whole lifetime of the subnebula.

Reactions (3) and (4) are illustrated by Fig. 5 which represents the conversion times of $\mathrm{CO}$ to $\mathrm{CH}_{4}$ and $\mathrm{N}_{2}$ to $\mathrm{NH}_{3}$ in our model of Saturn's subnebula. These chemical times are calculated from the data given by Prinn \& Barshay (1977), Lewis \& Prinn (1980), and Smith (1998), and using the temperature and pressure profiles derived from our subnebula model (Sect. 2.3). The results are presented at several epochs of the subnebula's life and at different distances from Saturn. Taking into account the kinetics of chemical reactions, one can infer that the efficiency of the conversion is limited only to the inner part of Saturn's subnebula and at early times of its evolution. Figure 6 shows the radii at which the conversion time equals the age of the disk as a function of disk age. Again, it shows that $\mathrm{CO}$ can be efficiently converted to $\mathrm{CH}_{4}$ only in the innermost zones of Saturn's subnebula (below $\sim 11 R_{\mathrm{Sat}}$ ). Similarly, $\mathrm{N}_{2}$ can be efficiently converted to $\mathrm{NH}_{3}$ below $\sim 7 R_{\text {Sat }}$. As a consequence, the products of reaction 3 and 4 cannot have been incorporated in planetesimals that took part in the formation of Titan (whose present location is $\sim 20 R_{\text {Sat }}$ ).

Our calculations imply that $\mathrm{CO}: \mathrm{CH}_{4}$ and $\mathrm{N}_{2}: \mathrm{NH}_{3}$ ratios remain almost constant during the whole lifetime of Saturn's subnebula. Moreover, since reaction (2) is showed to play no role in Saturn's subnebula, the $\mathrm{CO}_{2}$ : $\mathrm{CO}$ ratio also remains fixed during its lifetime. These conclusions are compatible with those found by MGB02 in the case of their colder turbulent model of Saturn's subnebula. This implies that, based on homogeneous gas phase chemistry, the $\mathrm{CO}_{2}: \mathrm{CO}: \mathrm{CH}_{4}$ and $\mathrm{N}_{2}: \mathrm{NH}_{3}$ ratios were close to the values initially acquired in Saturn's feeding zone.

\subsection{Heterogeneous gas-phase chemistry}

In this Section, we examine the role that Fischer-Tropsch catalysis may play in the subnebula gas-phase. Since Fischer-Tropsch catalysis converts $\mathrm{CO}$ or $\mathrm{CO}_{2}$ and $\mathrm{H}_{2}$ into $\mathrm{CH}_{4}$ on the surface of transition metals such as iron and nickel, it may affect the 


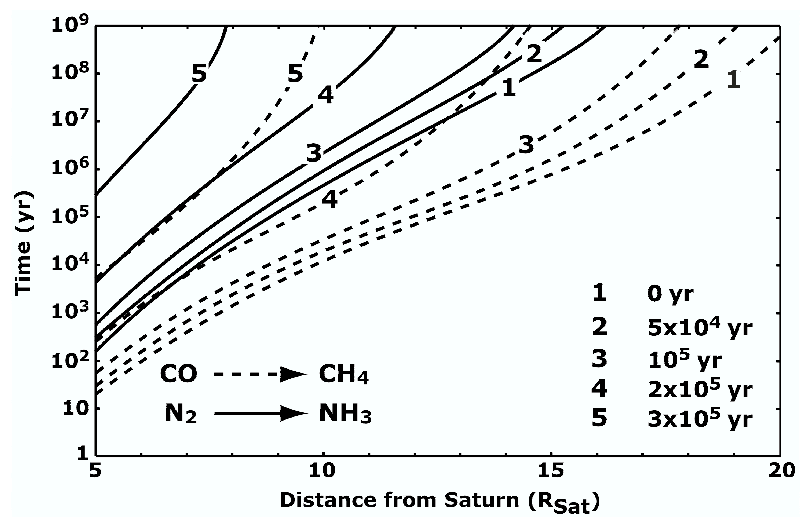

Fig. 5. Chemical time profiles calculated for $\mathrm{CO}: \mathrm{CH}_{4}$ and $\mathrm{N}_{2}: \mathrm{NH}_{3}$ conversions in our model of Saturn's subnebula. The conversion of $\mathrm{CO}$ to $\mathrm{CH}_{4}$ and of $\mathrm{N}_{2}$ to $\mathrm{NH}_{3}$ is fully inhibited, except quite close to Saturn and at the early times of the subnebula evolution.

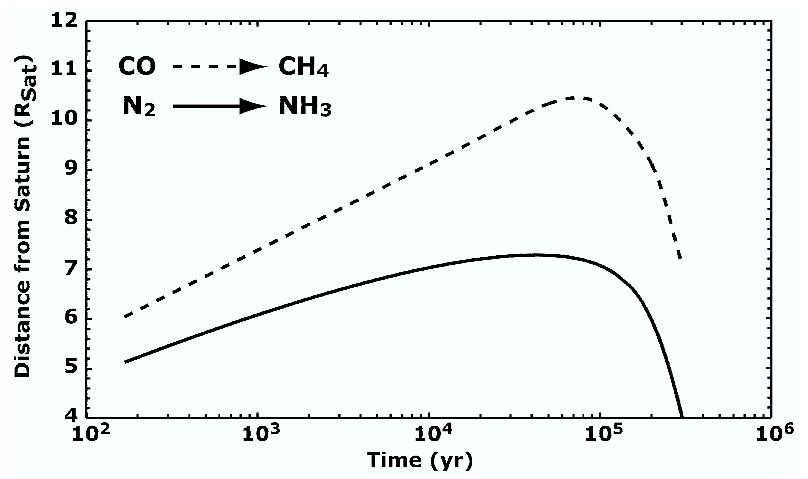

Fig. 6. Radii at which the chemical conversion times of $\mathrm{CO}$ to $\mathrm{CH}_{4}$ and of $\mathrm{N}_{2}$ to $\mathrm{NH}_{3}$ are equal to the disk's lifetime.

$\mathrm{CO}_{2}: \mathrm{CO}: \mathrm{CH}_{4}$ gas phase ratios in the early and warm Saturn subnebula.

In order to calculate the chemical times corresponding to Fischer-Tropsch catalysis, we use recent results obtained by Sekine et al. (2005) to which the reader is referred for further details. According to these laboratory experiments conducted at low pressures $\left(2 \times 10^{-2}-5 \times 10^{-1}\right.$ bar $)$ and with $\mathrm{H}_{2}: \mathrm{CO}$ and $\mathrm{H}_{2}: \mathrm{CO}_{2}$ gas-phase ratios of 1000 , conversions of $\mathrm{CO}$ into $\mathrm{CH}_{4}$ and $\mathrm{CO}_{2}$ into $\mathrm{CH}_{4}$ via Fischer-Tropsch catalysis proceed efficiently only in a narrow region in the subdisk, corresponding to a temperature of $\sim 550 \mathrm{~K}$. The experimental results by Sekine et al. (2005) show that the $\mathrm{CH}_{4}$ formation rates from $\mathrm{CO}$ and $\mathrm{CO}_{2}$ at $550 \mathrm{~K}$ both increase with pressure and show similar pressure dependence. The experimental data also show that the $\mathrm{CH}_{4}$ formation rates from $\mathrm{CO}_{2}$ are about 0.01 times those from $\mathrm{CO}$ under same reaction conditions.

Using the thermodynamic conditions derived in Sect. 2.3, we have calculated the timescales for conversions of $\mathrm{CO}$ into $\mathrm{CH}_{4}$ $\left(t_{\mathrm{CO} \rightarrow \mathrm{CH}_{4}}\right)$ and of $\mathrm{CO}_{2}$ into $\mathrm{CH}_{4}\left(t_{\mathrm{CO}_{2} \rightarrow \mathrm{CH}_{4}}\right)$ at $550 \mathrm{~K}$ as a function of the distance from Saturn. Note that since measurements by Sekine et al. (2005) were conducted in the $10^{-2}-10^{-1}$ bar pressure region, values at lower pressure range have been extrapolated using a linear fit. We also assume that about $10 \%$ of the cosmic component of iron is present as metal in the gas-phase (Pollack et al. 1994) and that the particles are spherical with a radius of $10^{-6} \mathrm{~m}$. The number densities of $\mathrm{CO}$ and $\mathrm{CO}_{2}$ are given by solar composition, assuming that all the carbon atoms are in the form of $\mathrm{CO}$ and $\mathrm{CO}_{2}$, respectively. This assumption gives

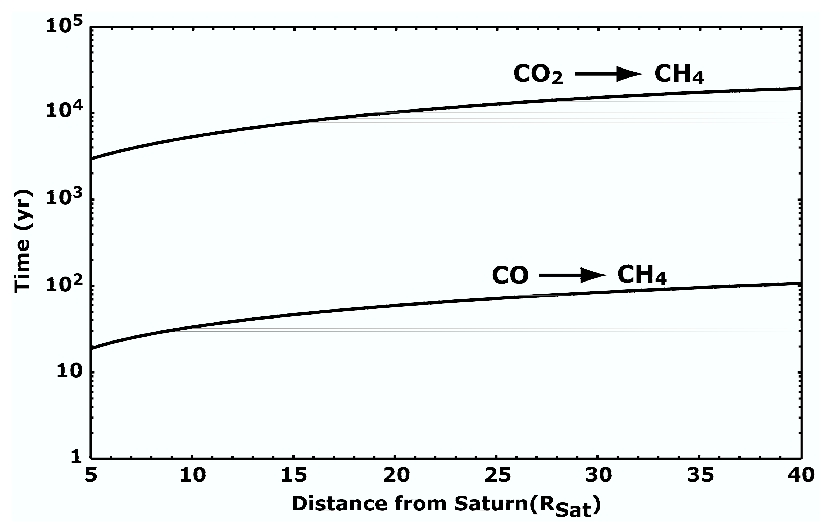

Fig. 7. Timescales for the conversions of $\mathrm{CO}$ into $\mathrm{CH}_{4}$ and of $\mathrm{CO}_{2}$ into $\mathrm{CH}_{4}$ at a temperature of $550 \mathrm{~K}$ in the subnebula as a function of the distance from Saturn.

an upper value of timescales for the conversions of $\mathrm{CO}$ and $\mathrm{CO}_{2}$ into $\mathrm{CH}_{4}$ in the subnebula.

Fischer-Tropsch catalysis is efficient up to the distance of $\sim 40 R_{\text {Sat }}$ since the temperature of $550 \mathrm{~K}$ is never reached at greater distances from Saturn. Figure 7 represents $t_{\mathrm{CO} \rightarrow \mathrm{CH}_{4}}$ and $t_{\mathrm{CO}_{2} \rightarrow \mathrm{CH}_{4}}$ calculated as a function of the distance from Saturn in the subnebula. Note that these chemical times are calculated at a given distance from Saturn only when the local temperature is equal to $550 \mathrm{~K}$. Therefore, the chemical timescales presented in Figs. 7 and 8 are calculated at an epoch varying with the distance from Saturn.

Figure 7 shows that the conversion times of $\mathrm{CO}$, and to a lesser extent $\mathrm{CO}_{2}$, into $\mathrm{CH}_{4}$ are very short. Therefore, it is likely that the chemical equilibrium is attained very rapidly in the inner region of Saturn's subnebula, and that the $\mathrm{CO}_{2}: \mathrm{CO}: \mathrm{CH}_{4}$ ratios in the gas phase are modified due to FischerTropsch catalysis compared to the values acquired from the solar nebula.

However, the next step is to know wether the products of Fischer-Tropsch catalysis may be trapped inside planetesimals produced in Saturn's subnebula. To address this question, we consider two timescales. The first one, $t_{\text {viscous }}$, is the viscous timescale of the gas, i.e. the time required for an element of the subnebula to be accreted by Saturn. This timescale is given by $t_{\text {viscous }}=2 r^{2} / 3 \tilde{v}$ where $r$ and $\tilde{v}$ are the considered distance to Saturn and the local viscosity in the subnebula, respectively. The second timescale, $t_{\text {cool }}$, is the time required for the subnebula to cool down from $550 \mathrm{~K}$ (the temperature at which Fischer-Tropsch catalysis is efficient) to the condensation temperature of water ice (taken to be $150 \mathrm{~K}$ in our calculations).

As shown by Fig. 8, $t_{\text {viscous }} \ll t_{\text {cool }}$ in the catalytically active region of the subnebula. Therefore, an element of gas is accreted onto Saturn before reaching a temperature low enough to trap the products of Fischer-Tropsch catalysis in ices ${ }^{2}$. Therefore, even if a catalytically active region may have existed in the early Saturn subnebula, it is likely that it has not affected the composition of volatiles trapped in the icy planetesimals produced in the subdisk at temperatures below $150 \mathrm{~K}$. This implies

2 Note moreover that 1 ) values of $t_{\text {cool }}$ should be higher than those used in this work since volatiles are trapped as hydrates or clathrate hydrates at lower temperatures than $150 \mathrm{~K}$ in Saturn's subnebula, and 2) $t_{\text {cool }}$ is calculated at a given distance to Saturn, whereas the gas in the subnebula migrates toward the inner (and hotter) part of the subnebula. The real time needed, for a given element of gas of the subnebula, to cool to $150 \mathrm{~K}$ is therefore longer than $t_{\text {cool }}$. 


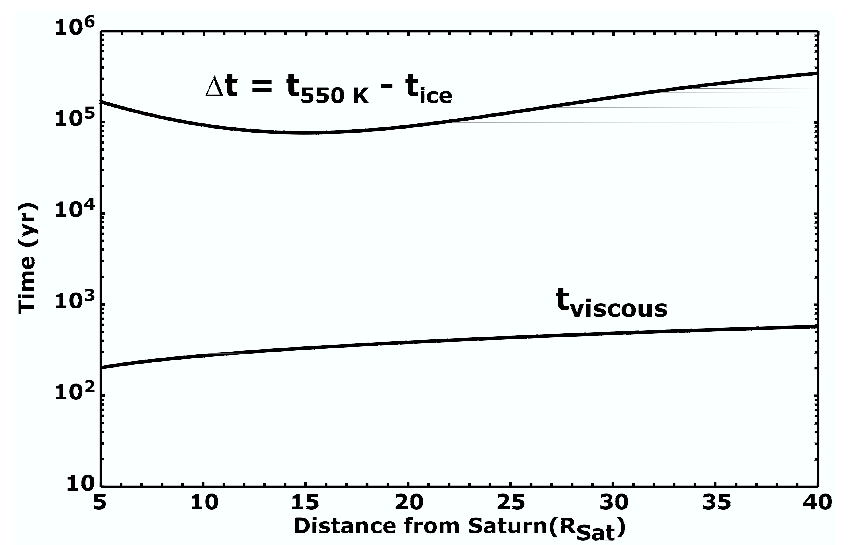

Fig. 8. From top to bottom: timescale needed by the subnebula to cool down from $550 \mathrm{~K}$ to the condensation temperature of water ice $(\sim 150 \mathrm{~K})$ and falling timescale of the subdisk's material as a function of the distance from Saturn when the local temperature is equal to $550 \mathrm{~K}$.

that the methane trapped in planetesimals produced in Saturn's subnebula originates from the solar nebula. As a consequence, Fischer-Tropsch catalysis in the subnebula gas-phase cannot be invoked to explain the present day abundance of methane in Titan's atmosphere.

\section{Composition of icy planetesimals}

Forming satellites grow from planetesimals that may originate from the solar nebula and/or Saturn's subnebula. Two extreme cases can then be considered. In the first case, solids are carried away by gas flowing from the nebula to the subnebula, and, if they survive vaporization, they may be incorporated in the satellites. In the second case, icy grains form directly in the cooling subnebula. They grow by agglomeration, decouple from the gas and ultimately form planetesimals.

In this section, we give an estimate of the primordial composition of the ices incorporated in planetesimals for the two extreme cases mentioned above (formation in the solar nebula or in the subnebula). In both cases, the composition of ices incorporated in planetesimals is calculated from the local thermodynamic conditions of the environment where they are assumed to be produced.

The composition of ices is determined using the clathrate hydrate trapping theory (Lunine \& Stevenson 1985), as presented e.g. in Alibert et al. (2005b) and Mousis et al. (2006a, hereafter MAB06). The clathration and hydratation processes result from the presence of crystalline water ice at the time of the trapping of volatiles in the formation zone of the planetesimals (subnebula or solar nebula). With time, the decrease of temperature and pressure conditions in the formation locations of planetesimals leads to the trapping of volatile species, as illustrated in Fig. 9, where we consider as an example the case of their formation in the solar nebula ${ }^{3}$. The trapping of volatiles is calculated using the stability curves of clathrate hydrates derived from the thermodynamic data of Lunine \& Stevenson (1985) and the evolutionary tracks of temperature and pressure at the heliocentric distance of 9.8 AU in the solar nebula (hereafter cooling curve), corresponding to the current position of Saturn. The stability curves of $\mathrm{CO}_{2}$ and $\mathrm{CO}$ pure condensates are derived from the

\footnotetext{
3 In case of the formation of planetesimals in Saturn's subnebula, we consider the cooling curves of the subnebula, and not that of the solar nebula.
}

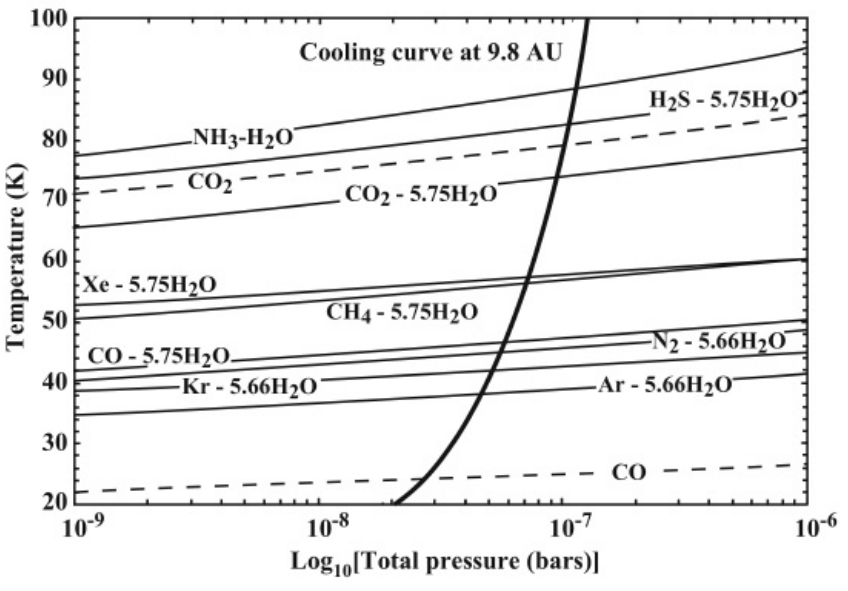

Fig. 9. Stability curves of the species trapped as hydrates or clathrate hydrates considered in this work (solid lines) and the evolutionary track of the solar nebula in $P-T$ space at the heliocentric distance of $9.8 \mathrm{AU}$. Abundances of various elements are solar, with $\mathrm{CO}_{2}: \mathrm{CO}: \mathrm{CH}_{4}=30: 10: 1$ and $\mathrm{N}_{2}: \mathrm{NH}_{3}=1$ in the vapor phase. The stability curves of $\mathrm{CO}_{2}$ and $\mathrm{CO}$ pure condensates are also represented (dashed lines). Species remain in the vapor phase as long as they stay in the domains located above the curves of stability.

existing experimental data. The gas phases abundances in the solar nebula of the different species are taken as in MAB06. In particular, we assume that $\mathrm{CO}_{2}: \mathrm{CO}: \mathrm{CH}_{4}=30: 10: 1$ and $\mathrm{N}_{2}: \mathrm{NH}_{3}=1$ as molar mixing ratios in the gas since they are considered as the nominal ones by MAB06 to interpret the enrichments in $\mathrm{C}$ and $\mathrm{N}$ (compared to solar) measured in Saturn. We then use them in all the calculations presented in this paper, for consistency.

The cooling curve intercepts the stability curves of the different ices at given temperature, pressure and surface density conditions. For each considered ice, the domain of stability is the region located below its corresponding stability curve. The stability curve of pure $\mathrm{CO}$ plays no role in our calculations when the abundance of water is assumed to be large enough to trap all the volatile species. Except for the unique case of $\mathrm{CO}_{2}{ }^{4}$, all species are assumed to be trapped under the form of hydrates or clathrate hydrates in the solar nebula gas-phase if the abundance of water is large enough.

The clathration and hydratation processes depend on the amount of water ice present in the trapping zone of volatiles (nebula or subnebula). In particular, it may stop if water ice is not abundant enough to trap the volatile species whose corresponding clathrate hydrates are stable at very low temperature in the solar nebula (those whose stability curves are located in the lower part of Fig. 9). For example, if one assumes O, C and $\mathrm{N}$ solar abundances in the solar nebula (Lodders 2003) with the afore mentioned $\mathrm{C}$ and $\mathrm{N}$ molar mixing ratios in the gas-phase, only $\mathrm{NH}_{3}, \mathrm{H}_{2} \mathrm{~S}$ and $\mathrm{Xe}$ can be entirely trapped by water, as hydrate of $\mathrm{NH}_{3}$ and clathrate hydrates of $\mathrm{H}_{2} \mathrm{~S}$ and $\mathrm{Xe}$ (we recall that $\mathrm{CO}_{2}$ is not trapped by water since it forms a pure condensate), and only roughly half of the $\mathrm{CH}_{4}$ existing in the gas-phase should be trapped as clathrate hydrate in the solar nebula. The remaining $\mathrm{CH}_{4}$, as well as the volatile species whose clathration processes occur at lower temperatures, remain in the vapor phase until the solar nebula cools enough to allow the formation of pure

\footnotetext{
4 From Fig. 9, it can be seen that $\mathrm{CO}_{2}$ crystallizes as a pure condensate prior to being trapped by water to form a clathrate hydrate during the cooling of the solar nebula. We then assume in this work that solid $\mathrm{CO}_{2}$ is the only existing form of $\mathrm{CO}_{2}$ condensate in the solar nebula.
} 
Table 2. Ratios of trapped masses of volatiles to the mass of $\mathrm{H}_{2} \mathrm{O}$ ice in planetesimals formed in Saturn's feeding zone in the solar nebula with $\mathrm{CO}_{2}: \mathrm{CO}: \mathrm{CH}_{4}=30: 10: 1$ and $\mathrm{N}_{2}: \mathrm{NH}_{3}=1$ in the gas-phase. The amount of available crystalline water ice is assumed to be large enough to trap all volatile species except $\mathrm{CO}_{2}$ (corresponding to $\mathrm{H}_{2} \mathrm{O}: \mathrm{H}_{2}=8.11 \times 10^{-4}$ in the gas-phase).

\begin{tabular}{ll}
\hline \hline Species & \\
\hline $\mathrm{CO}_{2}: \mathrm{H}_{2} \mathrm{O}$ & $8.91 \times 10^{-1}$ \\
$\mathrm{CO}: \mathrm{H}_{2} \mathrm{O}$ & $1.46 \times 10^{-1}$ \\
$\mathrm{CH}_{4}: \mathrm{H}_{2} \mathrm{O}$ & $9.14 \times 10^{-3}$ \\
$\mathrm{~N}_{2}: \mathrm{H}_{2} \mathrm{O}$ & $5.26 \times 10^{-2}$ \\
$\mathrm{NH}_{3}: \mathrm{H}_{2} \mathrm{O}$ & $4.67 \times 10^{-2}$ \\
$\mathrm{H}_{2} \mathrm{~S}: \mathrm{H}_{2} \mathrm{O}$ & $4.28 \times 10^{-2}$ \\
$\mathrm{Ar}: \mathrm{H}_{2} \mathrm{O}$ & $9.74 \times 10^{-3}$ \\
$\mathrm{Kr}: \mathrm{H}_{2} \mathrm{O}$ & $1.34 \times 10^{-5}$ \\
$\mathrm{Xe}: \mathrm{H}_{2} \mathrm{O}$ & $2.44 \times 10^{-6}$ \\
\hline
\end{tabular}

condensates (roughly between 20 and $30 \mathrm{~K}$ in the gas-phase conditions assumed for the solar nebula; see for example the stability curve of $\mathrm{CO}$ pure condensate in Fig. 9).

\subsection{Icy planetesimals produced in Saturn's feeding zone}

Assuming that the amount of available crystalline water ice is large enough to trap all volatile species except $\mathrm{CO}_{2}{ }^{5}$, MAB06 have calculated the composition of planetesimals formed in the solar nebula at the present location of Saturn. This allowed these authors to retrieve the abundance of carbon measured in Saturn by the Cassini spacecraft (Flasar et al. 2005) and to predict the abundances of N, S, Ar, Kr and Xe in its atmosphere. Moreover, under similar assumptions, Alibert et al. (2005b) were able to reproduce the abundances of $\mathrm{C}, \mathrm{N}, \mathrm{S}, \mathrm{Ar}, \mathrm{Kr}$ and Xe measured in situ by the Galileo probe within the upper atmosphere of Jupiter, in a way consistent with the formation and internal structure models of the giant planet. The nominal composition of ices incorporated in planetesimals formed in Saturn's feeding zone is reproduced in Table 2 .

\subsection{Icy planetesimals produced in Saturn's subnebula}

As shown in Fig. 4, ices carried away into the early Saturn's subnebula were all vaporized whatever the distance from the giant planet. With time, the subnebula cooled down and volatiles started to form condensates following a crystallization sequence similar to that described in the case of the solar nebula. Since, as shown in Sect. 3, homogeneous and heterogeneous gas-phase conversions were unable to affect the composition of the ices forming within the subdisk, the resulting volatile $\mathrm{i}$ to water mass ratio $\left(Y_{\mathrm{i}}\right)_{\text {sub }}$ in solids formed inside the subnebula can be expressed as a function of the initial one $\left(Y_{\mathrm{i}}\right)_{\text {feed }}$ in planetesimals produced in Saturn's feeding zone through the following relation:

$\left(Y_{\mathrm{i}}\right)_{\text {sub }}=f_{\mathrm{i}} \times\left(Y_{\mathrm{i}}\right)_{\text {feed }}$,

where $f_{\mathrm{i}}$ is the fractionation factor due to the consecutive vaporization and condensation of volatile $\mathrm{i}$ in the subdisk. Following the approach described by MA06, we have calculated that $f_{\mathrm{i}}$ approximatively ranges between 0.4 and 0.8 in Saturn's subnebula,

5 The required amount of water must be at least equal to $\mathrm{H}_{2} \mathrm{O}: \mathrm{H}_{2}=$ $8.11 \times 10^{-4}$, i.e. $\sim 4.9$ times the solar abundance. The over-solar abundance of water may result from sedimentation and drift of icy grains, due to gas drag (Supulver \& Lin 2000). as a function of the considered volatile species. For each species, the value of $f_{\mathrm{i}}$ is similar to that noted by MA06, in the case of the Jovian subnebula, where the reader is referred for further details. We therefore conclude that, whatever the formation location of planetesimals, and for a given abundance of water ice available for the clathration process, their composition does not strongly vary.

\section{Formation of Titan in Saturn's subnebula}

The origin of Titan is still uncertain. Although the thermochemical calculations, such as those presented in Sect. 4.1 (see Table 2), predict that carbon essentially exists in the form of $\mathrm{CO}$ and $\mathrm{CO}_{2}$ in planetesimals formed in the outer solar nebula, the main carbon species detected in the atmosphere of Titan is $\mathrm{CH}_{4}$, with a mole fraction of $4.92 \times 10^{-2}$ close to the surface (Niemann et al. 2005). The atmosphere of Titan is dominated by $\mathrm{N}_{2}$ and $\mathrm{CH}_{4}$, and $\mathrm{CO}$ is several orders of magnitude less abundant than $\mathrm{CH}_{4}\left(\mathrm{CO}: \mathrm{CH}_{4} \sim 10^{-3}\right.$; Gautier \& Raulin 1997). $\mathrm{CO}_{2}$ is also suspected to exist on the surface of Titan but its presence is not yet confirmed (Hartung et al. 2006). The existing nitrogen is presumed to be the product of ammonia photolysis or shock chemistry (Atreya et al. 1978; McKay et al. 1988) while the high deuterium to hydrogen $(\mathrm{D}: \mathrm{H})$ ratio observed in methane indicates a solar nebula origin for this compound (Mousis et al. $2002 \mathrm{~b}$ ). This latter hypothesis is in agreement with the plausible existence of a geological source for methane, consisting of a reservoir of clathrate hydrate in the interior of Titan, advocated by Tobie et al. (2006).

An unexpected feature of the atmosphere of Titan is that no noble gases other than argon have been detected by the Gas Chromatograph Mass Spectrometer (GCMS) aboard the Huygens probe during its descent. The detected argon includes primordial ${ }^{36} \mathrm{Ar}$ and the radiogenic isotope ${ }^{40} \mathrm{Ar}$, which is a decay product of ${ }^{40} \mathrm{~K}$ (Niemann et al. 2005). The other primordial noble gases ${ }^{38} \mathrm{Ar}, \mathrm{Kr}$ and Xe were not detected by the GCMS instrument, yielding an upper limit for their mole fractions of $10^{-8}$. ${ }^{36} \mathrm{Ar} /{ }^{14} \mathrm{~N}$ is about six orders of magnitude lower than the solar value, indicating that the amount of trapped ${ }^{36} \mathrm{Ar}$ is low within Titan (Niemann et al. 2005). The deficiency of Titan in noble gases is surprising since their abundances have been measured as over-solar in Jupiter (Owen et al. 1999), implying that they were provided by volatile-rich planetesimals during the formation of the giant planet (Alibert et al. 2005b). This latter conclusion is in agreement with our calculations in Sect. 4.1 of the composition of icy planetesimals produced in the solar nebula, at the present location of Saturn. Indeed, Table 2 shows that noble gases are incorporated in planetesimals produced at $\sim 10 \mathrm{AU}$, if the amount of crystalline water is large enough. Therefore, explaining both the lack of noble gases in Titan and their oversolar abundances in Jupiter's atmosphere is challenging.

We now investigate the different scenarios that could explain the deficiency of Titan in noble gases and CO. In each case, the explored scenarios invoke the formation of Titan from planetesimals that may contain volatiles under the form of hydrates, clathrate hydrates or pure condensates. These planetesimals can be $\mathrm{CO}_{2}$-rich since this species may exist in Titan (Hartung et al. 2006) and has even been detected on Phoebe (Clark et al. 2005), indicating that it was present in the solar nebula gas-phase (Phoebe is considered as a captured body that formed in the outer solar system - see Johnson \& Lunine 2005).

We first discuss the effect of a water deficiency in the formation zone of Saturn and Titan, inspired by a scenario proposed by Hersant et al. (2004, see also Hersant et al. 2005; hereafter H04 
and H05). In this scenario, Titan was formed in a cold subnebula, from planetesimals produced in the feeding zone of Saturn that have preserved their volatile content from vaporization during their migration/accretion in the subnebula. Moreover, in this scenario, the abundance of available water in Saturn's feeding zone is supposed to have been low enough to prevent the trapping of large amounts of $\mathrm{CO}, \mathrm{N}_{2}$ and noble gases as clathrate hydrates in the forming planetesimals. In our discussion, we point out the difficulties inherent to this hypothesis and we propose an alternative scenario in which Titan was formed from planetesimals that have suffered a partial vaporization during their migration in Saturn's subnebula. In both scenarios and following the model of the subnebula proposed in the present work, Titan was formed at different epochs of its evolution. In the first scenario derived from $\mathrm{H0} 4$ and $\mathrm{H} 05$, Titan was formed in a late and cold subneb$u l a$, corresponding to the phase 2 of our model. In the scenario we propose, Titan accreted planetesimals in a balmy subnebula, more precisely during the transition epoch from phase 1 to phase 2 when the temperature of the subnebula was high enough to vaporize $\mathrm{CO}, \mathrm{N}_{2}$, and the noble gases trapped in the drifting planetesimals, but too low to sublimate the other remaining ices.

\subsection{Formation in a late and cold subnebula}

Following the idea initially proposed by H04 and H05, we first assume that Titan formed in a late and cold subnebula, from planetesimals produced in the solar nebula and carried away by the gas flow toward the subnebula. In this scenario, the temperature was low enough to preserve from vaporization all the volatiles trapped in the planetesimals. Under this hypothesis, the composition of Titan should reflect that of the solids produced in Saturn's feeding zone, requiring that the composition of planetesimals accreted both by Saturn and Titan was similar.

We also assume, following $\mathrm{H} 04$ and H05, that the amount of water in the formation zone of the planetesimals was sufficiently high to allow the efficient clathration of $\mathrm{CH}_{4}$, but too low to allow that of $\mathrm{CO}$, as well as of $\mathrm{N}_{2}$ and noble gases. Indeed, such a choice of the abundance of water is possible, since the stability curve of the clathrate hydrates of $\mathrm{CO}$ is located at lower temperatures than that of $\mathrm{CH}_{4}$ (see Fig. 9) ${ }^{6}$. Ignoring any further condensation as pure ices, it is possible to conclude that $\mathrm{CO}, \mathrm{N}_{2}$ and noble gases were poorly incorporated by growing planetesimals and therefore by the forming Titan.

This first formation scenario implies that abundances of noble gases should be solar within Saturn's atmosphere if one ignores the crystallization of the more volatile species as pure

\footnotetext{
${ }^{6}$ We note that the stability curve of $\mathrm{Xe}$, as represented in Fig. 9, is slightly above that of $\mathrm{CH}_{4}$. If the clathration sequence stopped due to a lack of water after the trapping of $\mathrm{CH}_{4}, \mathrm{Xe}$ should be also efficiently trapped, and hence would be present in Titan. However, the stability curve of this species in the temperature range of interest $(\sim 60 \mathrm{~K})$ is highly uncertain. To our knowledge, there are no laboratory experiments reported at temperatures below $200 \mathrm{~K}$ for Xe (see the compilation of Lunine \& Stevenson 1985). Moreover, the relative positions of the two stability curves depend on the assumed $\mathrm{CH}_{4}$ and $\mathrm{Xe}$ abundances which may vary as a function of the considered gas-phase composition in the solar nebula (adopted compilation of elements, number of carbonated species and their relative abundances). Note finally that it has been proposed that clathrate hydrates formed from hydrocarbons and nitrogen on the surface of Titan are possibly a sink for the noble gases absent from its atmosphere (Osegovic \& Max 2005). Therefore, even if the clathration curve of Xe was indeed located at higher temperature than that of $\mathrm{CH}_{4}$, the trapping of this noble gas on the surface of Titan is not firmly excluded by the GCMS observations.
}

condensates in the giant planet's feeding zone. In addition, this scenario implies that the composition of planetesimals accreted by Saturn itself was different from that of planetesimals accreted by Jupiter (abundances of $\mathrm{Ar}, \mathrm{Kr}$ and Xe have been measured as over-solar in Jupiter; see Owen et al. 1999).

On the other hand, current scenarios of giant planets formation suggest that Jupiter and Saturn accreted planetesimals formed in very close regions of the solar system (between 5 and 10 AU for Jupiter, and 9 and 12 AU for Saturn, see Alibert et al. 2005c). Moreover, for a given gas-phase abundance and a given amount of available crystalline water, the composition of ices incorporated in planetesimals hardly depends on the distance from the Sun (Alibert et al. 2005b), implying that the composition of planetesimals accreted both by Jupiter and Saturn was similar. Therefore, this first scenario of Titan's formation requires strong variations of the abundance of water between the formation zones of Jupiter and Saturn: in the case of our adopted gas-phase composition, namely $\mathrm{CO}_{2}: \mathrm{CO}: \mathrm{CH}_{4}=30: 10: 1$ and $\mathrm{N}_{2}: \mathrm{NH}_{3}=1$, the abundance of $\mathrm{H}_{2} \mathrm{O}$ with respect to $\mathrm{H}_{2}$ required to explain the enrichments in volatiles measured in Jupiter (Alibert et al. $2005 b$ ) is $\sim 8 \times 10^{-4}$ in its feeding zone while it must not exceed $\sim 1.94 \times 10^{-4}$ in Saturn's feeding zone (value corresponding to a full trapping of only $\mathrm{NH}_{3}, \mathrm{H}_{2} \mathrm{~S}$ and $\mathrm{CH}_{4}$ ). The origin of such strong variations of the abundance of water ice, on small lengthscales, remains unclear.

In addition, as stated in Sect. 4, all species that could not be trapped as clathrate hydrates due to a deficiency of water remained in the vapor phase only until the local temperature has cooled down enough to allow their crystallization as a pure condensates. This is the case for $\mathrm{CO}$, whose crystallization temperature as pure condensate is $\sim 25 \mathrm{~K}$ (see Fig. 9). As a consequence, if $\mathrm{CH}_{4}$ was fully trapped as a clathrate hydrate and $\mathrm{CO}$ crystallized as a pure condensate, the resulting $\mathrm{CO}: \mathrm{CH}_{4}$ mass ratio in icy planetesimals produced in Saturn's feeding zone would be $\sim 10$ with $\mathrm{CO}_{2}: \mathrm{CO}: \mathrm{CH}_{4}=30: 10: 1$ and $\mathrm{N}_{2}: \mathrm{NH}_{3}=1$ in the solar nebula gas-phase. This, in turn, would give a molar mixing ratio of $\sim 6$ in Titan, a value orders of magnitude higher than that observed in its atmosphere $\left(\sim 10^{-3}\right.$; Gautier \& Raulin 1997). As a result, a deficiency of the available water in the formation region of Saturn, compared to Jupiter's feeding zone, cannot explain the low $\mathrm{CO}: \mathrm{CH}_{4}$ ratio observed in Titan.

As stated in Sect. 4.2, the composition of planetesimals formed in the subnebula does not strongly differ from that of planetesimals formed in the solar nebula. As a consequence, even if Titan formed from icy planetesimals produced in the subnebula, a deficiency of water in this environment cannot explain the low abundances of $\mathrm{CO}$ and noble gases in Titan.

\subsection{Formation in a (not so) early and balmy subnebula}

We now explore an alternative scenario: the formation of Titan in a balmy subnebula (corresponding to the transition epoch between phase 1 and phase 2 of the subnebula's evolution). During the whole subnebula evolution (during phase 1), 500 $M_{\text {Titan }}$ transited through the subdisk. Assuming a standard solid-to-gas ratio of $1 / 50$, this translates into $\sim 10 M_{\text {Titan }}$ of solids carryed away from the solar nebula to the subnebula. The actual amount of solids available to build the satellite depends on the fraction of these $10 M_{\text {Titan }}$ that grew fast enough to decouple from the gas. However, at the transition epoch between phase 1 and phase 2 , enough material is in principle available to build the satellite. Moreover, the subnebula is not a closed system, and larger scale solids decoupled from the gas phase and orbiting the Sun could be captured by the subnebula. 
In this scenario, Titan was formed from planetesimals either produced in the solar nebula and later transported within the subdisk or produced in the outer subnebula itself. In that case, planetesimals would have suffered a partial vaporization during their migration inside the subnebula. The migration of planetesimals in a balmy subnebula (as our model shows at intermediate epochs) would allow an efficient vaporization of most volatile species ( $\mathrm{CO}, \mathrm{N}_{2}$ and noble gases) whose clathration temperatures are low, whereas $\mathrm{H}_{2} \mathrm{O}, \mathrm{NH}_{3}, \mathrm{H}_{2} \mathrm{~S}, \mathrm{CO}_{2}$ and $\mathrm{CH}_{4}$ would remain incorporated in solids ${ }^{7}$.

This scenario does not require any deficiency of water ice in Saturn's feeding zone, compared to that of Jupiter, in order to explain the quasi-absence of noble gases observed in Titan. On the contrary, it can be assumed that the gas-phase conditions, including the abundance of water, were identical in both Saturn's and Jupiter's feeding zones. As a consequence, the abundance of noble gases in Saturn should be over-solar (see MAB06), similarly to Jupiter. On the other hand, the resulting $\mathrm{CO}: \mathrm{CH}_{4}$ ratio in Titan is difficult to estimate. It depends on the complex interplay between migration of planetesimals, vaporization and subnebula disk evolution. However, it would be lower than the primordial one if the temperatures encountered by planetesimals were high enough. Any possible capture of pure $\mathrm{CO}$ condensate in planetesimals would not have any consequences on this scenario since the condensation temperature of pure $\mathrm{CO}$ is lower than that of its associated clathrate hydrate. Therefore, any $\mathrm{CO}$ trapped in the form of a pure condensate would be vaporized due to the high temperatures encountered in the subnebula. Since, as stated in Sect. 4.2, the content of volatiles in solids produced in the subnebula is close in that of solids formed in the nebula, the origin of the planetesimals that took part to the formation of Titan remains difficult to constrain.

\section{Discussion and conclusions}

We have calculated a model of Saturn's subnebula using the formalism of AMB05 and MA06. This model is consistent with the last phases of Saturn formation, as derived from the model of Alibert et al. (2005c). As for the Jovian subnebula, Saturn's subnebula evolves in two phases. During phase 1, when the solar nebula has not yet dissipated, the subnebula is fed via its outer edge by gas and gas-coupled material originating from the solar nebula. The subnebula is in equilibrium and the accretion rate is given by the rate of gas accretion by the forming Saturn. When the solar nebula has dissipated, the subnebula enters to phase 2 of its evolution. It is progressively accreted by the forming Saturn, and expands, due to angular momentum conservation.

Using the thermodynamic conditions derived from our model, we have calculated the evolution of the $\mathrm{CO}_{2}: \mathrm{CO}: \mathrm{CH}_{4}$ and $\mathrm{N}_{2}: \mathrm{NH}_{3}$ molar mixing ratios in the gas phase of the subnebula. We have shown that these mixing ratios were not modified due to the thermodynamic conditions inside the subnebula. Moreover, our calculations show that, even if Fischer-Tropsch catalysis is likely to modify the $\mathrm{CO}_{2}: \mathrm{CO}: \mathrm{CH}_{4}$ ratios in a restricted area of Saturn's subnebula, it has no influence on the

7 Tiny amounts of $\mathrm{CO}, \mathrm{N}_{2}$ and noble gases may remain trapped in these planetesimals since multiple occupancy of a single cage of a given clathrate hydrate may occur (Lunine \& Stevenson 1985). In the present case, clathrate hydrates dominated by $\mathrm{H}_{2} \mathrm{~S}$ or $\mathrm{CH}_{4}$ may also incorporate some slight quantities of $\mathrm{CO}, \mathrm{N}_{2}$ and noble gases. This implies that, at a given pressure, the stability curve of a considered clathrate hydrate including a multiple guest occupancy would be at a temperature close to that of the pure clathrate hydrate of the most abundant guest compound. composition of volatiles trapped in the forming icy planetesimals. This results from the fact that all gas elements that have suffered Fischer-Tropsch catalysis are accreted by the forming planet without ever reaching a temperature low enough to condense volatiles in the form of hydrates, clathrate hydrates or pure ices. As a consequence, the presence of a catalytically active region in the subnebula implies that methane and water formed via Fischer-Tropsch catalysis are accreted with the gas onto the forming Saturn. This conclusion is similar to the one found by Mousis et al. (2006b) in the case of the Jovian subnebula. Finally, we conclude that the (homogeneous or heterogeneous) chemical processes that may occur in the subnebula are likely to have no effect on the composition of ices formed in the subnebula: the $\mathrm{CO}_{2}: \mathrm{CO}: \mathrm{CH}_{4}$ and $\mathrm{N}_{2}: \mathrm{NH}_{3}$ ratios in the zone of planetesimal formation (at temperatures lower than $150 \mathrm{~K}$ ) are the same as those acquired from the solar nebula.

We have explored two scenarios of Titan's formation. In the first scenario, inspired by $\mathrm{H} 04$ and H05, the formation of Titan occurs in a cold and late subnebula. Even assuming a low amount of water in the solar nebula, the trapping of $\mathrm{CO}$ in the form of pure condensate leads to a $\mathrm{CO}: \mathrm{CH}_{4}$ molar mixing ratio orders of magnitude above the value known in its atmosphere: a calculated CO:CH $4 \sim 6$ vs. a measured value of $\sim 10^{-3}$ (Gautier \& Raulin 1997). Moreover, this scenario seems difficult to reconcile with the trapping of noble gases in the close-by formation zone of Jupiter (Owen et al. 1999; Alibert et al. 2005b).

In the second scenario, Titan formed at earlier epochs, when the subnebula was warm enough to partly vaporize the planetesimals captured from the solar nebula. This results in the vaporization and loss of $\mathrm{CO}, \mathrm{Ar}, \mathrm{Kr}, \mathrm{N}_{2}$ and possibly $\mathrm{Xe}$. As a consequence, the forming Titan contained low amounts of $\mathrm{CO}$ and noble gases, as measured today in its atmosphere. This scenario does not require significant variations of water abundance between the formation zones of Jupiter and Saturn and allows to reproduce the Cassini measurements of carbon abundance in Saturn (Flasar et al. 2005). However, as discussed in MAB06, in this scenario, this recent determination of the abundance of $\mathrm{C}$ seems incompatible with the $\mathrm{N}$ abundance determined indirectly from the Earth (Briggs \& Sackett 1989). More calculations, taking into account migration of captured planetesimals, as well as their thermal evolution and vaporization in an evolving subnebula are necessary to quantitatively test this second formation scenario.

Our study does not bring any constraints on the formation region of planetesimals that took part to the formation of Titan and other regular satellites (planetesimals produced in the solar nebula, or formed in Saturn's subnebula). On the other hand, the measurement of the $\mathrm{D}: \mathrm{H}$ ratio in $\mathrm{H}_{2} \mathrm{O}$ ice on the surface of Titan could allow us to disentangle these two origins. Indeed, a D: $\mathrm{H}$ ratio in $\mathrm{H}_{2} \mathrm{O}$ close to the one measured in comets (highly enriched compared to the proto-solar value, see Altwegg \& Bockelée-Morvan 2003) would point toward an episode of isotopic exchange between $\mathrm{HDO}$ and $\mathrm{H}_{2}$ in the solar nebula, thus favoring a solar nebula origin of planetesimals. On the contrary, a D:H ratio substantially lower than the one measured in comets would result from a second isotopic exchange in the early phase of Saturn's subnebula. In fact, based on the subnebula model of MGB02 and in order to explain the value of D:H measured in the atmospheric methane of Titan $\left(8.7_{-1.9}^{+3.2} \times 10^{-5}\right.$, see Coustenis et al. 2003), Mousis et al. (2002b) have shown that this value could result from an isotopic exchange between $\mathrm{CH}_{3} \mathrm{D}$ and $\mathrm{H}_{2}$ in the solar nebula gas phase. However, these calculations, based on a cold subnebula model, did not take into account the possible vaporization of methane contained in planetesimal 
migration within the subnebula. In phase 1 of our subnebula model, methane can be vaporized, leading to an additional fractionation of the $\mathrm{D}: \mathrm{H}$ ratio in $\mathrm{CH}_{4}$ in planetesimals formed in situ, with respect to the one acquired by solids produced in the solar nebula. If planetesimals were produced in the subnebula, a gradient of D:H should exist both in methane and water in the satellites of Saturn. In particular, satellites formed close to Saturn should harbor a lower D:H ratio, compared to irregular satellites $^{8}$, or satellites formed at larger distances. Such calculations will be the subject of a future paper (Mousis \& Alibert 2007).

A possible test of the two formation scenarios (formation in a cold or a balmy subnebula) presented in this paper would be the measurement of noble gas abundances in the atmosphere of Saturn, since the first scenario predicts a solar abundance, whereas the second one implies an over-solar abundance. Moreover, as discussed in MAB06, new measurements of the nitrogen abundance in Saturn's atmosphere would also allow us to improve the determination of the $\mathrm{C}: \mathrm{N}$ ratio in the planet. This ratio depends in particular on the amount of water available in the formation zone of Saturn. Comparing this ratio and the one observed in Jupiter would put some strong constraints on the evolution of the primitive solar nebula, and on the formation of the two giant planets of our system. Future space missions may allow some of these measurements and shed new light on the formation of satellites and on processes at work in giant planet subnebulae.

Acknowledgements. This work was supported in part by the Swiss National Science Foundation.

\section{References}

Alibert, Y., Mordasini, C., \& Benz, W. 2004, A\&A, 417, L25

Alibert, Y., Mordasini, C., Benz, W., et al. 2005a, A\&A, 434, 343

Alibert, Y., Mousis, O., \& Benz, W. 2005b, ApJ, 622, L145

Alibert, Y., Mousis, O., Mordasini, C., et al. 2005c, ApJ, 626, L57

Alibert, Y., Mousis, O., \& Benz, W. 2005d, A\&A, 439, 1205 (AMB05)

Altwegg, K., \& Bockelée-Morvan, D. 2003, Space Sci. Rev., 106, 139

Atreya, S. K., Donahue, T. M., \& Kuhn, W. R. 1978, Science, 201, 611
Biemann, K., Lafleur, A. L., Owen, T., et al. 1976, Science, 194, 76 Briggs, F. H., \& Sackett, P. D. 1989, Icarus, 80, 77 Canup, R. M., \& Ward, W. R. 2002, AJ, 124, 3404

Canup, R. M., \& Ward, W. R. 2006, Nature, 441, 834

Clark, R. N., Brown, R. H., Jaumann, R., et al. 2005, Nature, 435, 66 Coradini, A., Federico, C., Forni, O., et al. 1995, Surv. Geophys., 16, 533

Coustenis, A., Salama, A., Schulz, B., et al. 2003, Icarus, 161, 383 Donahue, T. M., Hoffman, J. H., \& Hodges, R. R. 1981, GeoRL, 8, 513 Flasar, F. M., Achterberg, R. K., Conrath, B. J., et al. 2005, Science, 307, 1247 Gautier, D., \& Raulin, F. 1997, in Huygens: Science, Payload and Mission, ESA SP-1177, 359

Hartung, M., Herbst, T. M., Dumas, C., et al. 2006, JGR, 111, 7

Hersant, F., Gautier, D., \& Lunine, J. I. 2004, P\&SS, 52, 623 (H04)

Hersant, F., Gautier, D., Lunine, J. I., \& Tobie, G. 2005, DPS, 37, 675 (H05)

Johnson, T. V., \& Lunine, J. I. 2005, Nature, 435, 69

Lewis, J. S., \& Prinn, R. G. 1980, ApJ, 238, 357

Lodders, K. 2003, ApJ, 591, 1220

Lubow, S. H., Seibert, M., \& Artymowicz, P. 1999, ApJ, 526, 1001

Lunine, J. I., \& Stevenson, D. J. 1985, ApJS, 58, 493

Marty, B., Heber, V. S., Grimberg, A., et al. 2006, M\&PS, 41, 739

McKay, C. P., Scattergood, T. W., Pollack, J. B., et al. 1988, Nature, 332, 520

Magni, G., \& Coradini, A. 2004, Planet. Space Sci., 52, 343

Mousis, O., \& Alibert, Y. 2006, A\&A, 448, 771 (MA06)

Mousis, O., \& Alibert, Y. 2007, in prep.

Mousis, O., Gautier, D., \& Bockelée-Morvan, D. 2002a, Icarus, 156, 162 (MGB02)

Mousis, O., Gautier, D., \& Coustenis, A. 2002b, Icarus, 159, 156

Mousis, O., Alibert, Y., \& Benz, W. 2006a, A\&A, 449,411 (MAB06)

Mousis, O., Alibert, Y., Sekine, Y., et al. 2006b, A\&A, 459, 965

Niemann, H. B., Atreya, S. K., Bauer, S. J., et al. 2005, Nature, 438, 779

Osegovic, J. P., \& Max, M. D. 2005, JGRE, 110, 8004

Owen, T. C., Mahaffy, P. R., Niemann, H. B., et al. 1999, Nature, 402, 269

Papaloizou, J. C. B., \& Terquem, C. 1999, ApJ, 521, 823

Pollack, J. B., Hollenbach, D., Beckwith, S., et al. 1994, ApJ, 421, 615

Prinn, R. G., \& Barshay, S. S. 1977, Science, 198, 1031

Prinn, R. G., \& Fegley Jr., B. 1981, ApJ, 249, 308

Prinn, R. G., \& Fegley Jr., B. 1989, in Origin and Evolution of Planetary and Satellites Atmospheres, ed. S. K. Atreya, J. B. Pollack, \& M. S. Matthews (Tucson: The University of Arizona Press), 78

Sekine, Y., Sugita, S., Shido, T., et al. 2005, Icarus, 178, 154

Shakura, N. I., \& Sunyaev, R. A. 1973, A\&A, 24, 337

Smith, M. D. 1998, Icarus, 132, 176

Stevenson, D. 2001, Science, 294, 71

Supulver, K. D., \& Lin, D. N. C. 2000, Icarus, 146, 525

Talbi, D., \& Herbst, E. 2002, A\&A, 386, 1139

Tobie, G., Lunine, J. I., \& Sotin, C. 2006, Nature, 440, 61

\footnotetext{
${ }^{8}$ Irregular satellites, like Phoebe, are believed to be formed in the solar nebula, and later captured by Saturn.
} 